\title{
Capacidade antioxidante de flores de capuchinha (Tropaeolum majus L.).
}

\section{Antioxidant capacity of nasturtium (Tropaeolum majus L.) flowers.}

\author{
Harícia de Almeida Souza ${ }^{1}$; Amanda Laís Alves Almeida Nascimento ${ }^{2}$; Paulo César \\ Stringheta $^{3}$; Frederico Augusto Ribeiro de Barros ${ }^{4}$
}

\begin{abstract}
RESUMO: As flores comestíveis são utilizadas na alimentação humana há centenas de anos a fim de melhorar a aparência e o sabor de diversos pratos na culinária. Dentre as flores que podem ser ingeridas, uma das mais conhecidas é a capuchinha (Tropaeolum majus L.), de origem mexicana e peruana, cuja coloração do cálice varia, podendo ser amarelo claro, alaranjado ou vermelha. A capuchinha é fonte de compostos bioativos, como a quercetina, as antocianinas, o ácido gálico, os ácidos clorogénicos e carotenoides, os quais conferem capacidade antioxidante a essas flores. Assim, transcendendo a sua aparência visual e o seu sabor, a capuchinha oferece benefícios à saúde da população devido aos seus compostos antioxidantes, que ajudam no combate de doenças crônicas, auxiliam na prevenção de degeneração muscular, de desordens imunológicas e da oxidação de lipoproteínas de baixa densidade. Tendo isso em vista, o objetivo do trabalho foi determinar a capacidade antioxidante das flores de capuchinha. As flores utilizadas foram as capuchinhas alaranjadas, amarelas e vermelhas. A determinação da capacidade antioxidante foi feita através do método ABTS. As flores de capuchinha vermelha apresentaram a maior capacidade antioxidante $(13,59 \mu \mathrm{mol}$ de Trolox/g) seguido pelas flores alaranjadas $(4,10 \mu \mathrm{mol}$ de Trolox/g) e amarelas $(2,99$ $\mu \mathrm{mol}$ de Trolox/g). Este resultado pode ser explicado pela maior concentração de compostos fenólicos presentes nas flores vermelhas. Dessa forma, as capuchinhas, em especial as de flores vermelhas, poderiam ser mais utilizadas na alimentação humana devido aos seus potenciais benefícios para a saúde.
\end{abstract}

PALAVRAS CHAVE: Flores comestíveis; Compostos fenólicos; Carotenoides

ABSTRACT: Edible flowers have been used in human food for hundreds of years in order to improve the appearance and taste of different dishes. Among the edible flowers, nasturtium (Tropaeolum majus L.) is one of the most well-known, of Mexican and Peruvian origin, whose color varies, and may be light yellow, orange or red. Nasturtium is a source of bioactive compounds, such as quercetin, anthocyanins, gallic acid,

\footnotetext{
${ }^{1}$ Bolsista da FAPEMIG Bic-Júnior/2018-2019 do CAp-COLUNI. Atualmente, cursa a graduação em Engenharia Química na EEL-USP - e-mail: haricia@usp.br

${ }^{2}$ Estudante de doutorado do Departamento de Tecnologia de Alimentos da Universidade Federal de Viçosa - e-mail: amandalais00@hotmail.com

${ }^{3}$ Professor colaborador- Departamento de Tecnologia de Alimentos da Universidade Federal de Viçosa e-mail: stringap@ufv.br

${ }^{4}$ Professor Orientador- Departamento de Tecnologia de Alimentos da Universidade Federal de Viçosa e-mail: fredbarros@ufv.br

Agradecemos a FAPEMIG pela bolsa Bic-Júnior e incentivo à pesquisa
}

COLÉGIO DE APLICAÇÃO-COLUNI / UNIVERSIDADE FEDERAL DE VIÇOSA

https://periodicos.ufv.br/rpv 
chlorogenic acids and carotenoids, which provide antioxidant capacity to these flowers. Thus, besides its visual appearance and flavor, nasturtium flowers may provide health benefits to the population due to their antioxidant compounds, which help to fight chronic diseases, to prevent muscle degeneration, immunological disorders and oxidation of low density lipoproteins. The objective of this work was to determine the antioxidant capacity of nasturtium flowers (orange, yellow and red). The antioxidant capacity was determined using the ABTS method. Red nasturtium flowers showed the highest antioxidant capacity $(13.59 \mu \mathrm{mol}$ of Trolox/g) followed by orange $(4.10 \mu \mathrm{mol}$ of Trolox/g) and yellow $(2.99 \mu \mathrm{mol}$ of Trolox/g). This result can be explained by the higher concentration of phenolic compounds present in red flowers. Thus, nasturtium, especially those with red flowers, could be used more often in human nutrition due to their potential health benefits.

KEYWORDS: Edible flowers; Phenolic compounds; Carotenoids

\section{INTRODUÇÃO.}

A capuchinha (Tropaeolum majus L.) é uma flor originária do México e do Peru. Com tonalidades que variam desde o amarelo claro até o vermelho, passando pelo laranja, a capuchinha é uma flor comestível bastante utilizada na culinária. Além disso, essa hortaliça não convencional, como as flores comestíveis são conhecidas, é uma fonte de compostos com atividade antioxidante, a exemplo dos carotenoides e dos compostos fenólicos.

As propriedades bioativas de flores comestíveis já foram investigadas em diversos trabalhos, nos quais se destacaram a presença de carotenoides e compostos fenólicos, responsáveis também pela coloração predominante em cada espécie (FRIEDMAN et al., 2010; BENVENUTI, BORTOLOTTI, MAGGINI, 2016).

Dessa forma, o consumo de capuchinha pode trazer diversos benefícios à saúde principalmente no combate aos radicais livres produzidos pelo metabolismo humano, evitando doenças oriundas do estresse oxidativo como obesidade, diabetes, doenças cardiovasculares e o câncer (FERNANDES et al. 2017; MELO et al, 2018).

\section{REVISÃO DE LITERATURA.}

\section{CAPUCHINHA.}

Existem diversas flores comestíveis, por exemplo, a couve-flor (Brassica olerace var. bortrytis), a alcachofra (Cynara cardunculus), o gerânio [Pelargonium 
hortorum (L.)], a cravina [Dianthus chinensis (L.)], a capuchinha (Tropaeolum majus L.), dentre outras. Essas hortaliças não convencionais, como são conhecidas, são amplamente utilizadas na culinária desde tempos remotos. Há evidências históricas de que eram utilizadas pelos romanos como alimento, assim como também pelos chineses e povos do Médio Oriente (NEWMAN E O'CONNER, 2009).

A capuchinha possui um sabor apimentado, podendo ser utilizada para substituir a mostarda em saladas, é bastante usada no preparo de empadas e omeletes. Além disso, a sua coloração variada, do amarelo claro ao vermelho, incluindo o alaranjado, oferece uma diversidade de coloração aos pratos que compõem. Contudo, algumas são incorporadas em vinhos e licores aromatizados, sendo que as flores maiores, como a de abóbora, podem ainda ser recheadas e fritas (FELIPPE, 2004).

As capuchinhas são ricas em luteína, antocianinas (cianidina, delfinidina e pelargonidina), benzilglucosinolato além de possuírem uma significativa quantidade de vitamina C. A mesma também é usada em algumas receitas e práticas medicamentosas como formas alternativas de medicação com um custo menor e maior acessibilidade (DEMATTI \& COAN, 2004; FONT QUER, 1993; CORRÊA, 1926).

A capacidade fotoprotetora do extrato de flores de capuchinha foi comprovada em um estudo realizado por Bazylko et al. (2014). Este estudo mostrou que o extrato foi capaz de inibir espécies reativas de oxigênio (ERO) produzidas por radiações UVA e UVB. Os autores relatam que esta planta é utilizada para tratamento dermatológico de assaduras, queimaduras solares e descamação (caspa) o que pode ser atribuído a sua capacidade fotoprotetora (BAZYLKO et al., 2014).

Um recente estudo avaliou também a capacidade antiadipogênica in vitro do extrato da capuchinha. Foi observado que o extrato foi capaz de inibir o acúmulo lipídico e reduzir a expressão gênica de fatores que aumentam o processo de adipogênese. Os autores ressaltam que a capuchinha apresenta potencial terapêutico inovador para a prevenção e tratamento de obesidade (KIM et al., 2017).

As folhas de capuchinha também demonstraram potencial medicinal, em estudos conduzidos com camundongos, sendo que o extrato das folhas apresentou atividade diurética, que auxilia também no tratamento de infecções urinárias efeito antihipertensivo e anticoagulante (SANTO et al., 2007; GASPAROTTO JUNIOR et al., 2009, 2011, 2012). 
As flores comestíveis são usadas principalmente na decoração de pratos na gastronomia. Entretanto, os benefícios dessas flores não se limitam aos seus atributos visuais e sensoriais, pois a riqueza de compostos bioativos confere às flores comestíveis diversos benefícios a saúde.

O cultivo de flores comestíveis é diferenciado dos outros vegetais, pois é realizado sem o uso de pesticidas, em um ambiente protegido com o fornecimento controlado de luz e água. Desse modo, as hortaliças não convencionais não podem ser adquiridas em qualquer estabelecimento, além de ter espécies, como a azaleia, o lírio e o copo-de-leite, que não podem ser consumidas por conterem compostos tóxicos.

\section{METABOLISMO VEGETAL.}

Os vegetais em geral possuem dois metabólitos, o primário e o secundário. Os primários são responsáveis pela sobrevivência da planta, sendo ativo em três processos principais: a respiração, a fotossíntese e a assimilação de nutrientes. O metabolismo secundário está relacionado às estratégias de defesa das plantas e aos compostos antioxidantes produzidos.

O metabolismo secundário não é essencial para o ciclo de vida das plantas, mas desempenha diversos papeis importantes nos vegetais. Um exemplo disso é a proteção que o metabólito secundário oferece a estresses abióticos, como mudanças de temperatura, disponibilidade de água e minerais, exposição a luz solar e a UV.

Além dessas vantagens, há estudos que demonstram a importâncias dos compostos fenólicos, produzidos pelo metabolismo secundário, na conquista definitiva do ambiente terrestre pelas plantas, como a lignina, por exemplo, que conferiu rigidez aos vasos, possibilitando o desenvolvimento do sistema vascular vegetal.

O metabólito secundário tem importância para duas áreas em especial: a nutracêutica e a fitomedicina. A primeira responsável por estudar os compostos fitoquímicos presentes em cereais, vegetais e frutas, e o segundo voltado ao estudo das aplicações das plantas medicinais na cura de diversas doenças.

Os carotenoides são pigmentos naturais que, conjuntamente com a clorofila, participa da fotossíntese realizada pelo metabolismo primário. Ademais, os carotenoides têm atividade antioxidante, pró-vitamina A e lipofílico, desempenhando a função, dessa forma, antioxidante sobre as proteínas HDL ("High Density Lipoproteins") e LDL (“Low Density Lipoproteins”). 


\section{CAPACIDADE ANTIOXIDANTE.}

A capacidade antioxidante da capuchinha é caracterizada, principalmente, pelos carotenoides (Figura 1) e pelos compostos fenólicos presentes nela, como a quercetina (Figura 2), o ácido gálico (Figura 3), as antocianinas (Figura 4), dentre outros.

Figura 1: Estruturas moleculares de três carotenoides presentes em capuchinhas.<smiles>CC1=C(/C=C/C(C)=C/C=C/C(C)=C/C=C/C=C(C)/C=C/C=C(C)/C=C/C=C(C)/C=C/C2=C(C)CCCC2(C)C)C(C)(C)CCC1</smiles>

Betacaroteno<smiles>CC1=C(/C=C/C(C)=C/C=C/C(C)=C/C=C/C=C(C)/C=C/C=C(C)/C=C/C2=C(C)CC(O)CC2(C)C)C(C)(C)CC(O)C1</smiles>

Zeaxantina<smiles>CC1=CC(O)CC(C)(C)C1/C=C/C(C)=C/C=C/C(C)=C/C=C/C=C(C)/C=C/C=C(C)/C=C/C1=C(C)CC(O)CC1(C)C</smiles>

Luteína

Fonte: SILVA et al., 2010.

Figura 2: Estrutura molecular da quercetina<smiles>O=c1c(O)c(-c2ccc(O)c(O)c2)oc2cc(O)cc(O)c12</smiles>

Fonte: SIMÕES et al., 2013 
Figura 3: Estrutura molecular do ácido gálico

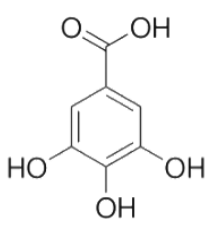

Fonte: SOUZA FILHO et al., 2006

Figura 4: Molécula de antocianidina, estrutura principal das antocianinas.<smiles>[R]c1cc(-c2[o+]c3cc(O)cc(O)c3cc2O)cc([R])c1O</smiles>

Fonte: SILVA et al., 2010.

A capacidade antioxidante pode ser expressa de vários modos: pela quantificação de produtos originados durante a peroxidação de lipídeos (TBARS, a oxidação do LDL, co-oxidação do $\beta$-caroteno), CUPRAC (cupric ion reducing antioxidant capacity), pela eliminação de um radical peroxil (ORAC - oxygen radical absorbance capacity, TRAP - total reactive antioxidant potential), pela capacidade de eliminação de um radical orgânico (ABTS - 2,20-azino-bis (ácido 3ethylbenzthiazoline-6-sulfônico), DPPH (peroxidação do 2,2-difenil-1-picrylhydrazil), dentre outros.

Os métodos mais utilizados para a determinação da capacidade antioxidante in vitro são: FRAP (do inglês, Ferric Reducing Antioxidant Power), ABTS (ácido 2,2azino-bis(3-etilbenzotiazolin)-6-sulfônico) e DPPH (2,2-difenil-1-picril-hidrazila) e ORAC (do inglês, Oxygen Radical Absorbance Capacity). No presente trabalho, será utilizado o método ABTS para analisar a atividade antioxidante da capuchinha a fim de avaliar os efeitos que a luz LED ocasiona na capacidade antioxidante dessas flores comestíveis. 


\section{OBJETIVOS.}

O trabalho teve como objetivo determinar a capacidade antioxidante de capuchinhas alaranjadas, amarelas e vermelhas.

\section{MATERIAIS E MÉTODOS.}

A semeadura e cultivo das capuchinhas (Tropaeolum majus L.) foram realizados no setor de Floricultura, do Departamento de Fitotecnia da Universidade Federal de Viçosa em Viçosa - MG (Latitude 2045' Sul, Logitude 42 ${ }^{\circ}$ 52' Oeste, Altitude $642 \mathrm{~m}$ ).

Para obtenção das mudas foram utilizadas sementes sem tratamento químico da marca ISLA. As sementes foram distribuídas em bandeja de isopor contendo substrato de cultivo comercial. Após 15 dias, quando apresentavam aproximadamente $10 \mathrm{~cm}$ de altura, foram transplantadas para vasos de $10 \mathrm{~L}$ contendo substrato inicialmente fertilizado com nitrogênio, fosforo e potássio (4:14:8) na proporção de $1 \mathrm{~g}$ para cada 1 $\mathrm{kg}$ de substrato. As plantas foram regadas diariamente conforme necessário e o experimento foi realizado em casa de vegetação. As flores foram coletadas e utilizadas inteiras e in natura (Figura 6).

Figura 6: Capuchinhas utilizadas no estudo

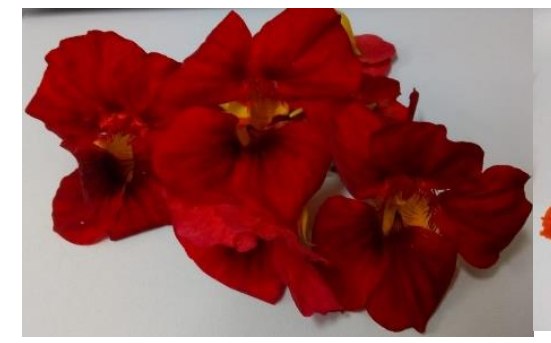

Capuchinha vermelha

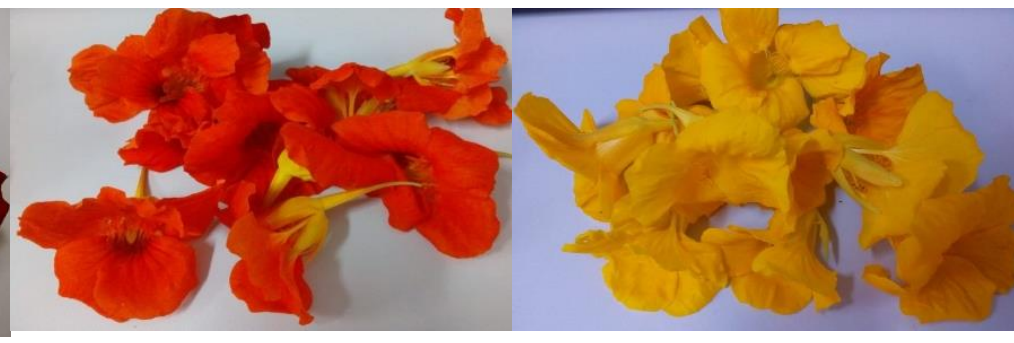

Capuchinha alaranjada
Capuchinha amarela

Fonte: Dos autores, 2019

As análises de capacidade antioxidante foram realizadas no Laboratório de Corantes Naturais e Compostos Bioativos (LaCBio) do Departamento de Tecnologia de Alimentos da Universidade Federal de Viçosa. O extrato fenólico foi obtido por meio da maceração das flores, inteiras e in natura, em etanol $70 \%$ acidificado ( $\mathrm{pH} \mathrm{2,0 \pm 0,1)}$ 
pela adição de solução de $\mathrm{HCl}$ concentrado. A extração foi realizada em banho ultrassônico (Ultracleaner 1400A, Unique, Brasil) $40 \mathrm{kHz}$ a $25^{\circ} \mathrm{C}$ por $60 \mathrm{~min}$. Os extratos foram filtrados e o volume aferido em balão volumétrico. Os extratos fenólicos obtidos estão apresentados na figura abaixo (Figura 7) e o processo de obtenção desses extratos está representado no fluxograma da Figura 8.

Figura 7- Extratos fenólicos das flores de capuchinhas

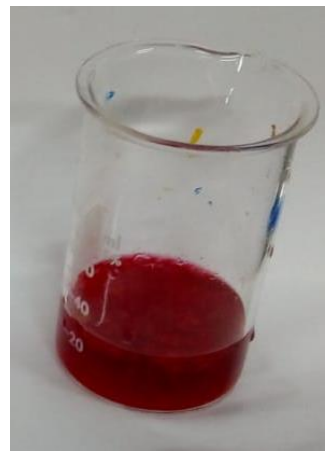

Capuchinha vermelha

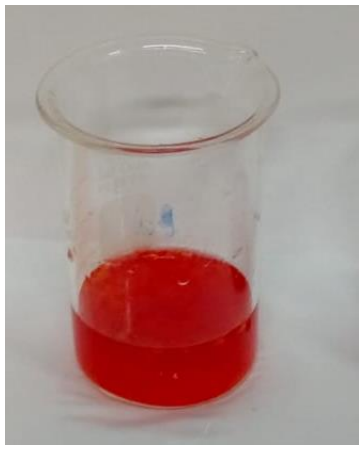

Capuchinha alaranjada

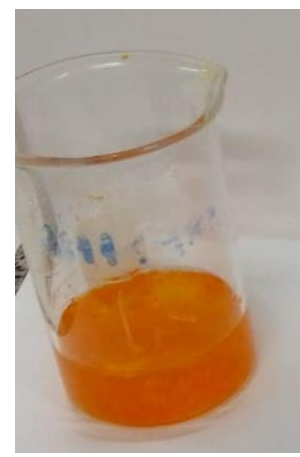

Capuchinha amarela

Fonte: Dos autores, 2019

Figura 8- Fluxograma das etapas de obtenção dos extratos fenólicos das flores de capuchinha.

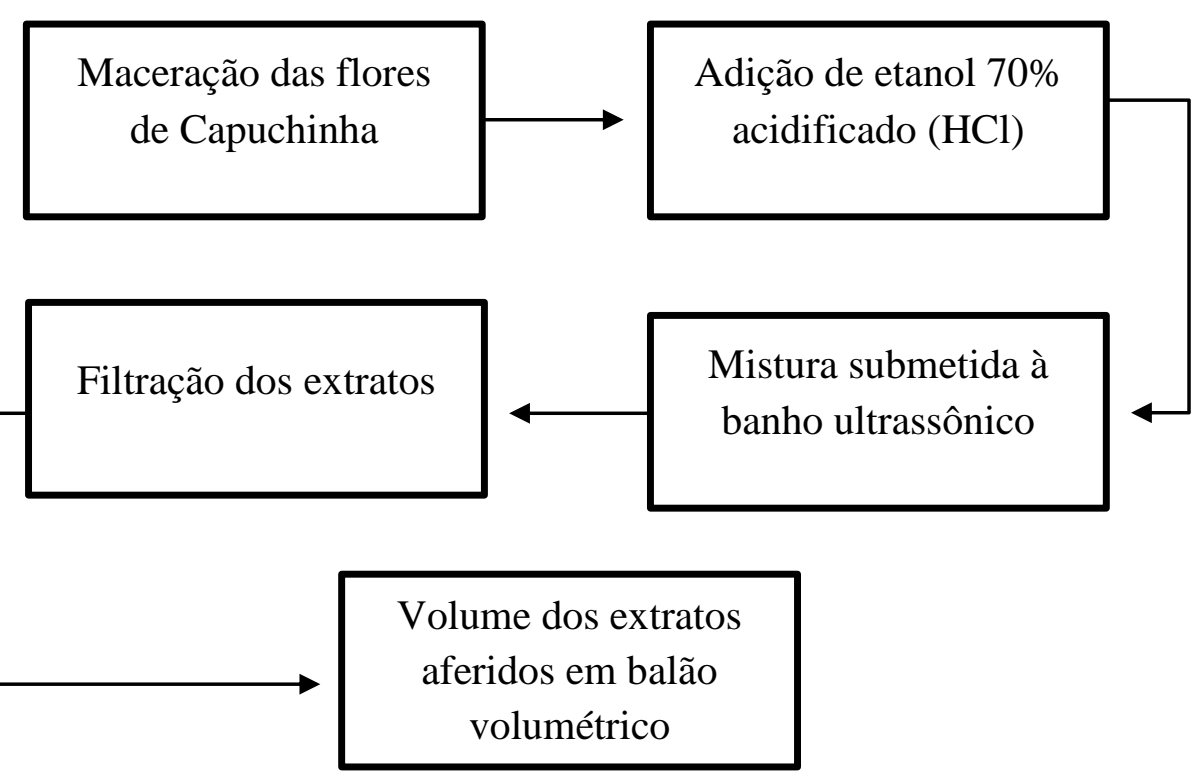

Fonte: Dos autores, 2019 
O preparo do radical foi feito adicionando à solução de ABTS•+ (ácido 2,2azino-bis(3-etilbenzotiazolin)-6-sulfônico) na concentração de $7 \mathrm{mmol} . \mathrm{L}^{-1}$, uma solução de persulfato de sódio $\left(2,45 \mathrm{mmol} . \mathrm{L}^{-1}\right)$ na proporção $1: 1$. Em 3,5 $\mathrm{mL}$ da solução radical ABTS $\bullet+$ foram adicionados $0,5 \mathrm{~mL}$ de cada extrato, sendo que a leitura espectrofotométrica foi feita após 6 minutos de reação (RE et al., 1999). Foi realizada uma curva padrão de Trolox e os resultados foram expressos em equivalente de Trolox $\left(\mu \mathrm{mol}\right.$ Trolox.g $\left.{ }^{-1}\right)$. A equação dessa curva obtida é dada pela Equação 1, cujo coeficiente de relação foi de 0,974 .

$$
y=-0,005 x+0,606
$$

onde $\mathrm{y}=$ Absorvância e $\mathrm{x}=$ concentração de Trolox $(\mu \mathrm{M})$

$\mathrm{O}$ experimento foi realizado em 3 repetições. Os dados foram submetidos à análise de variância (ANOVA), teste Tukey, ao nível de 5\% de significância. Todas as análises estatísticas foram realizadas no software R:R versão 3.6.0.

\section{RESULTADOS E DISCUSSÃO.}

Ao analisar a capacidade antioxidante das capuchinhas (Tabela 1), foi possível observar diferenças entre os valores obtidos das amostras. Nota-se que a capuchinha vermelha apresentou a maior capacidade antioxidante. Nossos resultados estão de acordo com outros estudos (RONCHETI, 2018; NASCIMENTO, 2019). A capacidade antioxidante de flores de capuchinha vermelha é geralmente maior pois elas possuem maior concentração de antocianinas, principalmente a pelargonidina-3-glicosídeo, entre outros fenólicos tais como ácidos fenólicos e quercetina (GARZÓN \& WROLSTAD, 2009; RONCHETI, 2018; NASCIMENTO, 2019). Esses compostos fenólicos estão diretamente relacionados com o poder antioxidante dos vegetais e atuam na prevenção de diversas doenças crônicas.

As flores amarelas possuem maiores teores de carotenoides totais, sendo que a luteína é o único carotenoide encontrado em capuchinhas, e seu consumo está relacionado à saúde ocular (LI et al. 2014). As capuchinhas alaranjadas possuem teores intermediários de fenólicos e carotenoides quando comparadas às flores amarelas e vermelhas. 
Os resultados também podem ser explicados baseado na extração utilizada. A metodologia de extração, utilizando etanol $70 \%$ acidificado como solvente, possui maior afinidade por compostos fenólicos (ex: antocianinas) que estão em maior concentração na capuchinha vermelha. As capuchinhas amarelas e alaranjadas possuem uma quantidade maior de carotenoides que são compostos lipofílicos e, dessa forma, são extraídos em menor quantidade.

Tabela 1: Capacidade antioxidante das Capuchinhas

\begin{tabular}{cc} 
Cor das Capuchinhas & $\begin{array}{c}\text { Capacidade antioxidante }(\boldsymbol{\mu m o l} \text { de Trolox } / g \text { de } \\
\text { amostra) }\end{array}$ \\
\hline Alaranjada & $4,10 \pm 0,15^{\mathrm{a}}$ \\
Amarela & $2,99 \pm 0,20^{\mathrm{a}}$ \\
Vermelha & $13,59 \pm 0,55^{\mathrm{b}}$ \\
\hline
\end{tabular}

Médias seguidas de mesma letra não diferem estatisticamente entre si, a 5\% de probabilidade, pelo teste de Tukey; $\quad \pm$ desvio padrão.

Fonte: Dados dos autores

\section{CONCLUSÃO.}

Pode-se concluir que os três tipos de capuchinhas, amarelas, alaranjadas e vermelhas, podem trazer benefícios à saúde devido a confirmação de atividade antioxidante dessas flores comestíveis. Além disso, as flores de capuchinha vermelha são as que possuem o maior potencial antioxidante, comprovado neste estudo.

\section{REFERÊNCIAS BIBLIOGRÁFICAS.}

BAZYLKO, A. et al. Inhibition of ROS production, photoprotection, and total phenolic, flavonoids and ascorbic acid content of fresh herb juice and extracts from the leaves and flowers of Tropaeolum majus. Industrial Crops and Products, v. 55, p. 19-24, 1 abr. 2014.

BENVENUTI, S.; BORTOLOTTI, E.; MAGGINI, R. Antioxidant power, anthocyanin content and organoleptic performance of edible flowers. Scientia Horticulturae, v. 199, p. 170-177, 16 fev. 2016. 
CORRÊA MP. Dicionário das plantas úteis do Brasil e das exóticas cultivadas. Rio de Janeiro: Ministério da Agricultura e Instituto Brasileiro de Desenvolvimento Florestal. v. 1. 1926.

DEMATTI MESP; COAN RM. Jardins com plantas medicinais. Jaboticabal: FUNEP. p.274, 2004.

FELIPPE, G.M. Entre o jardim e a horta: as flores que vão para a mesa. $2 .^{\text {a }}$ edição. São Paulo: Senac. 2004.

FERNANDES, L. et al. Edible flowers: A review of the nutritional, antioxidant, antimicrobial properties and effects on human health. Journal of Food Composition and Analysis, v. 60, p. 38-50, 1 jul. 2017.

FONT QUER P. Plantas medicinales: el dioscórides renovado. Barcelona: Editorial Labor S.A., Tomo II, 637p. 1993.

FRIEDMAN, H. et al. Characterization of yield, sensitivity to Botrytis cinerea and antioxidant content of several rose species suitable for edible flowers. Scientia Horticulturae, v. 123, n. 3, p. 395-401, 4 jan. 2010.

GARZÓN, G. A.; WROLSTAD, R. E. Major anthocyanins and antioxidant activity of Nasturtium flowers (Tropaeolum majus). Food Chemistry, v. 114, p. 44-49, 2009.

GASPAROTTO JUNIOR, A. et al. Antihypertensive effects of isoquercitrin and extracts from Tropaeolum majus L.: Evidence for the inhibition of angiotensin converting enzyme. Journal of Ethnopharmacology, v. 134, n. 2, p. 363-372, 24 mar. 2011.

GASPAROTTO JUNIOR, A. et al. Mechanisms underlying the diuretic effects of Tropaeolum majus L. extracts and its main component isoquercitrin. Journal of Ethnopharmacology, v. 141, n. 1, p. 501-509, 7 maio 2012.

GASPAROTTO JUNIOR, A. et al. Natriuretic and diuretic effects of Tropaeolum majus (Tropaeolaceae) in rats. Journal of Ethnopharmacology, v. 122, n. 3, p. 517522, 21 abr. 2009.

GOBBO-NETO, L., \& LOPES, N. P. Plantas medicinais: fatores de influência no conteúdo de metabólitos secundários. Química Nova, 30(2), 374-381. 2007.

LI, B. et al. Inactivity of human $\beta, \beta$-carotene-9',10'-dioxygenase (BCO2) underlies retinal accumulation of the human macular carotenoid pigment. Proceedings of the National Academy of Sciences of the United States of America, v. 111(28), p.10173-8, 2014.

KIM, G.-C. et al. Anti-adipogenic effects of Tropaeolum majus (nasturtium) ethanol extract on 3T3-L1 cells. Food \& Nutrition Research, v. 61, n. 1, p. 1-8, 14 jan. 2017. 
MELO, M. N. DE O. et al. Phenolic compounds from Viscum album tinctures enhanced antitumor activity in melanoma murine cancer cells. Saudi Pharmaceutical Journal, v. 26, n. 3, p. 311-322, 1 mar. 2018.

NASCIMENTO, A.L.A.A. Efeito de diferentes tempos de exposição à luz led vermelha em compostos bioativos de flores de capuchinha (tropaeolum majus L.)Dissertação (mestrado). Universidade Federal de Viçosa, 2019, 70p.

NEWMAN, S.E., \& O'CONNER, A.S. Edible Flowers. CSU Extension; n. 7237. http://www.ext.colostate.edu/ pubs/garden/07237.html. 2009.

RE, R., PELleGrini, N., PROTEGGENTE, A., PANNAlA, A., YANG, M., \& RICE-EVANS, C. Antioxidant activity applying an improved ABTS radical cation decolorization assay. Free Radical Biology \& Medicine, 1999.

RONCHETI, E.F.S. Efeito da radiação solar e da suplementação de luz led na biossíntese de compostos bioativos em flores de capuchinha (tropaeolum majus L.). Tese (doutorado). Universidade Federal de Viçosa, 2018, 92p.

SANTOS, A. P. E. et al. Efeito anticoagulante in vitro do extrato hidroetanólico de folhas e flores édulas de Tropaeolum majus L. (Tropaeolaceae) sobre o plasma humano. Latin American Journal of Pharmacy, v. 26, nº 5, 2007.

SIlVA, M. L. C., COSTA, R. S., SANTANA, A. S., \& KOBlitZ, M. G. B. Compostos fenólicos, carotenóides e atividade antioxidante em produtos vegetais. Semina: Ciências Agrárias, Londrina, v. 31, n. 3, p. 669-682. 2010.

SIMÕES, V. N., et al. Síntese, caracterização e estudo das propriedades de um novo complexo mononuclear contendo quercetina e íon Ga (III). Química Nova, 36(4), 495-501. 2013.

SOUZA FILHO et al. Potencial alelopático de Myrcia guianensis. Planta Daninha, 24(4), 649-656. 2006. 